\title{
The maximum piercing number for some classes of convex sets with the $(4,3)$-property
}

\author{
Jan Kynčl Martin Tancer \\ Department of Applied Mathematics and Institute for Theoretical Computer Science (ITI)* \\ Charles University, Malostranské nám. 25, 11800 Prague, Czech Republic \\ $\{$ kyncl, tancer\}@kam.mff.cuni.cz \\ Submitted: Sep 12, 2007; Accepted: Jan 30, 2008; Published: Feb 4, 2008 \\ Mathematics Subject Classification: 52A35
}

\begin{abstract}
A finite collection $\mathcal{C}$ of closed convex sets in $\mathbb{R}^{d}$ is said to have a $(p, q)$-property if among any $p$ members of $\mathcal{C}$ some $q$ have a non-empty intersection, and $|\mathcal{C}| \geq p$. A piercing number of $\mathcal{C}$ is defined as the minimal number $k$ such that there exists a $k$-element set which intersects every member of $\mathcal{C}$. We focus on the simplest non-trivial case in $\mathbb{R}^{2}$, i.e., $p=4$ and $q=3$. It is known that the maximum possible piercing number of a finite collection of closed convex sets in the plane with $(4,3)$-property is at least 3 and at most 13 . We consider the following three special types of collections of closed convex sets: segments in $\mathbb{R}^{d}$, unit discs in the plane and positively homothetic triangles in the plane, in each case only those satisfying $(4,3)$-property. We prove that the maximum possible piercing number is 2 for the collections of segments and 3 for the collections of the other two types.
\end{abstract}

\section{Introduction}

A finite collection $\mathcal{C}$ of closed convex sets in $d$-dimensional Euclidean space is said to have a $(p, q)$-property if among any $p$ members of $\mathcal{C}$ some $q$ have a non-empty intersection, and $|\mathcal{C}| \geq p$. A piercing number of $\mathcal{C}$ is defined as the minimal number $k$ such that there exists a $k$-element set which intersects every member of $\mathcal{C}$.

The well-known Helly Theorem [9] states that the piercing number of any finite collection of closed convex sets in $\mathbb{R}^{d}$ with $(d+1, d+1)$-property is equal to 1 . By considering a collection of hyperplanes in $\mathbb{R}^{d}$ in general position, we get that for $q \leq d$ the piercing number is unbounded. Hadwiger and Debrunner [6] conjectured that for every $p \geq q \geq d+1$

\footnotetext{
*ITI is supported by project 1M0545 of the Czech Ministry of Education.
} 

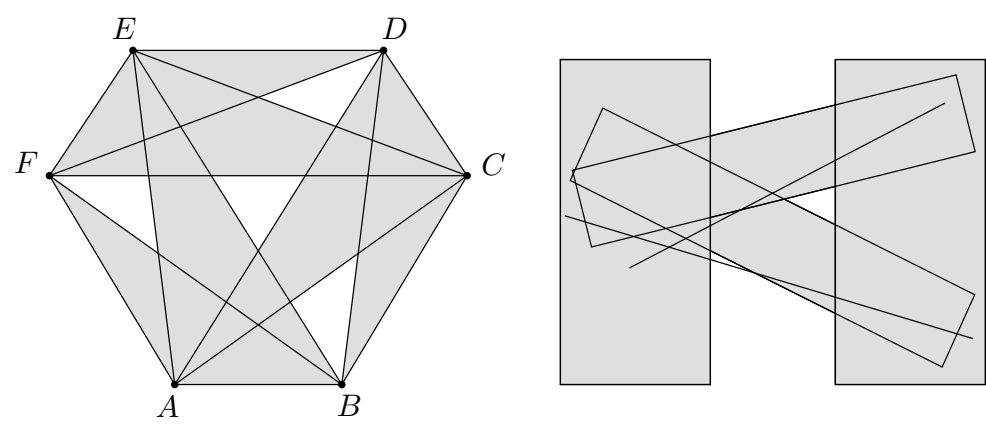

Figure 1: Collections of six sets with $(4,3)$-property and the piercing number 3. Left: six triangles $A C D, B C D, C E F, D E F, E A B, F A B$. Right: four rectangles and two segments, two of the rectangles are disjoint.

the piercing number of any finite collection of closed convex sets in $\mathbb{R}^{d}$ with $(p, q)$-property is bounded by a constant, which depends only on the values of $p, q$ and $d$. This conjecture has been proved by Alon and Kleitman [1,2].

Now, we can ask the following question: what is the exact value of $M(p, q ; d)$, the maximum possible piercing number of a finite collection of closed convex sets in $\mathbb{R}^{d}$ with $(p, q)$-property? The general arguments $[1,2]$ do not give any reasonable upper bounds on $M(p, q ; d)$. The simplest non-trivial case of this problem occurs for $p=4, q=3$ and $d=2$. It is easy to construct a collection of six triangles in the plane with $(4,3)$-property and the piercing number 3 (for an example, see Figure 1, left). There also exists such collection with two disjoint sets; see Figure 1, right. In this paper we construct two more examples with another restrictions on the involved sets. But a collection with the piercing number 4 has not been found yet. The best known upper bound, $M(4,3 ; 2) \leq 13$, has been established by Kleitman, Gyárfás and Tóth [11].

It seems to be quite difficult to improve these bounds significantly. So, we approach the problem from a different direction: we try to find the exact value of the maximum possible piercing number for some restricted collections of closed convex sets which satisfy $(4,3)$-property. In particular, we consider collections of segments in $\mathbb{R}^{d}$, unit discs in the plane and positively homothetic triangles in the plane. We prove that the maximum possible piercing number is equal to 2 for segments and 3 for discs and triangles. Similar results were proved for $d$-dimensional boxes in $\mathbb{R}^{d}$ with edges parallel to the coordinate axes [7,8]; it is known that for $2 \leq q \leq p \leq 2 q-2$ the maximum piercing number is $p-q+1$. It is also known that the maximum piercing number is 6 for the collections of unit discs satisfying $(3,2)$-property [13], 4 for the collections of pairwise intersecting discs (i.e., with $(2,2)$-property) [3], and at most 3 for the collections of pairwise intersecting translates of a convex set [10]. See the survey by Eckhoff [4] for other related results. The survey [4] also refers to the paper of Wegner [13], who claims that the piercing number of the collection of unit discs with $(4,3)$-property is 3 , but he does not give a proof of that. He only states that one can prove it similarly as the upper bound for the case of $(3,2)$-property, but it may be not so easy. So we believe it is worth giving the proof in this paper. 


\section{Preliminaries}

In this section we introduce notation and some propositions used throughout the paper.

Let $\mathbb{R}^{d}$ be a $d$-dimensional Euclidean space. Let $x \in \mathbb{R}^{d}$ and $S \subseteq \mathbb{R}^{d}$. We will say that $x$ pierces $S$ if $x \in S$.

Let $\mathcal{C}$ be a collection of subsets of $\mathbb{R}^{d}$ and let $X$ be a set of points in $\mathbb{R}^{d}$. We will say that $X$ pierces $\mathcal{C}$ if every $C \in \mathcal{C}$ is intersected by $X$.

For a finite collection $\mathcal{S}$ of sets define $P(\mathcal{S})$ as the piercing number of $S$, i. e.,

$$
P(\mathcal{S})=\min \{|X| ; X \text { pierces } \mathcal{S}\} .
$$

Now for a collection $\mathcal{C}$ define

$$
M_{\mathcal{C}}=\max \{P(\mathcal{S}) \mid \mathcal{S} \text { is a finite subcollection of } \mathcal{C} \text { with the }(4,3) \text {-property }\} .
$$

Our aim is to determine precise values of $M_{\mathcal{C}}$ for the three collections $\mathcal{C}$ mentioned in the introduction.

Now we formulate Helly's Theorem [9], and then introduce two lemmas widely used throughout the paper.

Theorem 1 (Helly). Let $d \geq 1$ be an integer and let $\mathcal{C}=\left\{C_{1}, C_{2}, \ldots, C_{k}\right\}, k \geq d+1$, be a collection of convex subsets of $\mathbb{R}^{d}$. If the intersection of every $d+1$ elements of $\mathcal{C}$ is non-empty, then the intersection of all the elements of $\mathcal{C}$ is non-empty.

Lemma 2. Let $\mathcal{S}$ be a collection of subsets of $\mathbb{R}^{d}$ satisfying $(4,3)$-property.

1. There are no three pairwise disjoint sets $S_{1}, S_{2}, S_{3} \in \mathcal{S}$.

2. If $A, B \in \mathcal{S}$ are two disjoint sets, then one of these sets intersects all the sets from $\mathcal{S} \backslash\{A, B\}$.

Proof.

1. For contradiction, suppose that there exist pairwise disjoint sets $S_{1}, S_{2}, S_{3} \in \mathcal{S}$. According to (4,3)-property, $|\mathcal{S}| \geq 4$, so there exists $S \in \mathcal{S} \backslash\left\{S_{1}, S_{2}, S_{3}\right\}$. By using $(4,3)$-property for the quadruple $S_{1}, S_{2}, S_{3}, S$ we obtain a contradiction.

2. For contradiction, suppose that there exist $C_{1}, C_{2} \in \mathcal{S} \backslash\{A, B\}$ such that $C_{1} \cap A=\emptyset$ and $C_{2} \cap B=\emptyset$. According to the first part of this lemma, $C_{1} \neq C_{2}$. But then $(4,3)$-property is not satisfied for $A, B, C_{1}, C_{2}$.

Let $S \subseteq \mathbb{R}^{2}$ and $v \in \mathbb{R}^{2}$. We will use the notation $-S=\{-s \mid s \in S\}$, and $S+v=$ $\{s+v \mid s \in S\}$.

Lemma 3. Let $S \subseteq \mathbb{R}^{2}$ be an arbitrary set and let $v_{1}, v_{2}, \ldots, v_{k} \in \mathbb{R}^{2}$. Then the sets $S+v_{1}, S+v_{2}, \ldots, S+v_{k}$ have a non-empty intersection if and only if there exists $v \in \mathbb{R}^{2}$ such that the set $-S+v$ contains all the points $v_{1}, v_{2}, \ldots, v_{n}$.

Proof. The statement easily follows from the following equivalence: $v \in S+v_{i} \Leftrightarrow-v \in$ $-S-v_{i} \Leftrightarrow v_{i} \in-S+v$. 

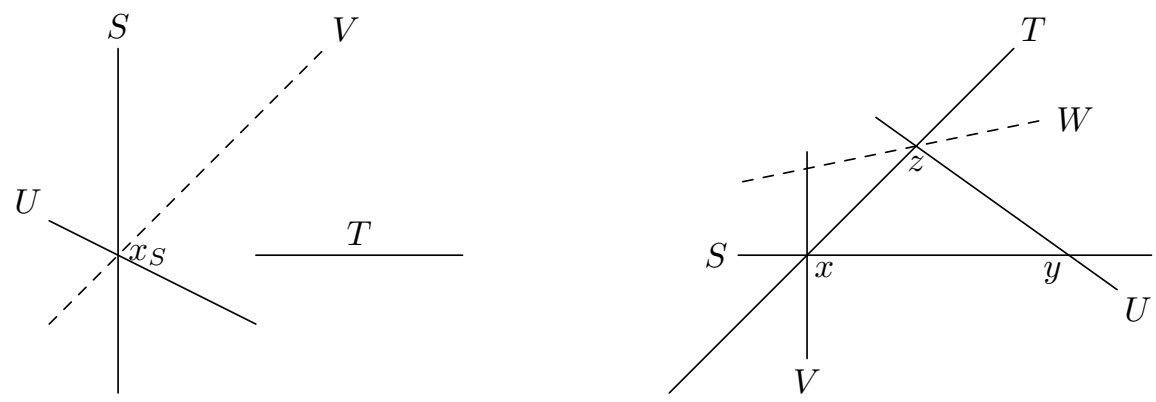

Figure 2: No two segments lie on a common line.

\section{Segments}

Let $\mathcal{G}_{d}$ be a collection of all segments in $\mathbb{R}^{d}$.

Theorem 4. $M_{\mathcal{G}_{d}}=2$.

Proof. To show that $M_{\mathcal{G}_{d}} \geq 2$, consider a collection of four segments where three of them have a common point and are disjoint with the fourth segment.

For the second inequality, let $\mathcal{S}$ be a finite subcollection of $\mathcal{G}_{d}$ satisfying (4,3)-property. We will show that $P(\mathcal{S}) \leq 2$.

In this proof, a segment will always mean a segment which is an element of $\mathcal{S}$.

We distinguish several cases:

1. No two segments lie on a common line.

See Figure 2, left, for the first of the following subcases, and right, for the second one.

1.1. There exist two disjoint segments $S, T$.

Let $U \in \mathcal{S}$ be any segment different from $S$ and $T$. By Lemma 2 we observe that $S \cap U \neq \emptyset$ or $T \cap U \neq \emptyset$. Note that $|S \cap U| \leq 1$ and $|T \cap U| \leq 1$, since there are no two segments lying on a common line. If $S \cap U \neq \emptyset$, let $\left\{x_{S}\right\}=S \cap U$, otherwise choose $x_{S}$ as an arbitrary point of $S$, similarly we choose a point $x_{T}$. According to the first observation, the segments $S, T$ and $U$ are pierced by the points $x_{S}$ and $x_{T}$. Let $V$ be a segment different from $S, T$ and $U$. Then among the segments $S, T, U, V$ there is a triple with non-empty intersection. Since $S \cap T=\emptyset$, we have $S \cap U \cap V=\emptyset$ or $T \cap U \cap V=\emptyset$, hence $x_{S} \in V$ or $x_{T} \in V$. Therefore, points $x_{S}$ and $x_{T}$ pierce $\mathcal{S}$.

1.2. Every two segments intersect.

If $S_{1} \cap S_{2} \cap S_{3} \neq \emptyset$ for each triple of segments $S_{1}, S_{2}, S_{3}$, then by Helly's Theorem, one point is sufficient to pierce all the segments.

So we can assume that there are three segments $S, T, U$ such that $S \cap T \cap U=\emptyset$. According to the assumptions for this subcase, we have $|S \cap T|=|S \cap U|=$ $|T \cap U|=1$. Let $V \in \mathcal{S}, V \neq S, T, U$. Among the segments $S, T, U, V$ there 
is a triple with non-empty intersection. This triple is different from the triple $S, T, U$. Without loss of generality, we can assume that from the remaining triples, $S, T, V$ is the triple with non-empty intersection. Since $|S \cap T|=1$, we have $|S \cap T \cap V|=1$. Let $S \cap T \cap V=\{x\}$ and $S \cap U=\{y\}$. Then $S, T$, $U$ and $V$ are pierced by $x, y$. It remains to show that every other segment $W$ from $\mathcal{S}$ contains $x$ or $y$. Actually, we show that every such $W$ contains $x$.

Let $T \cap U=\{z\}$. Note that $x \neq y \neq z \neq x$, because $S \cap T \cap U=\emptyset$. From the (4,3)-property for the segments $S, T, U, W$ we get that $W$ contains at least one of the points $x, y, z$. Moreover, $W$ does not contain two of them, according to the assumptions for this case. For contradiction, suppose that $x \notin W$. By symmetry, we can assume that $z \in W$ and $y \notin W$, so $W \cap U=\{z\}$. Then, among the segments $S, V, U, W$, no three have a common point. This violates the (4,3)-property: $S \cap V=\{x\}$, but $x \notin W \cup U$; similarly $W \cap U=\{z\}$, but $z \notin S \cup V$. As a corollary we obtain that $\{x, y\}$ pierces $\mathcal{S}$.

2. Two of the segments lie on a common line.

2.1. There exist two disjoint segments on a common line $p$.

2.1.1. All the segments lie on $p$.

Consider $p$ being a horizontal line. Let $L \in M$ be a segment whose right endpoint $x_{l}$ is the leftmost point among all right endpoints of the segments. Similarly, let $R$ be a segment whose left endpoint $x_{r}$ is the rightmost point among all left endpoints of the segments. Every other segment $S$ intersects $L$ or $R$. By the definition of $x_{l}$ and $x_{r}, S$ must contain at least one of these two points.

2.1.2. There exists a segment $S \nsubseteq p$.

Let $T_{1}$ and $T_{2}$ be two disjoint segments on $p$. By Lemma $2, S$ must intersect exactly one of these two segments, say $T_{1}$. Let $\{x\}=S \cap T_{1}$. By $(4,3)$-property, every other segment $T$ (different from $S, T_{1}, T_{2}$ ) must pass through $x$. Thus we can choose the second piercing point as an arbitrary point from $T_{2}$.

2.2. Every two segments lying on a common line intersect.

2.2.1. There are two disjoint segments $S, T$.

$S$ and $T$ do not lie on a common line. Let $s$ and $t$ be the lines containing segments $S$ and $T$. If every segment lies on $s$ or $t$, then every two segments lying on $s$ intersect. Hence, according to the one-dimensional Helly's Theorem, there exists a point $x_{s} \in s$ which is contained in all these segments. Similarly we can find a point $x_{t} \in t$ which is contained in all the remaining segments lying on $t$. In case there exists a segment which does not lie on any of the two lines $s, t$, we can choose the required two points the same way as in subcase 1.1 . 


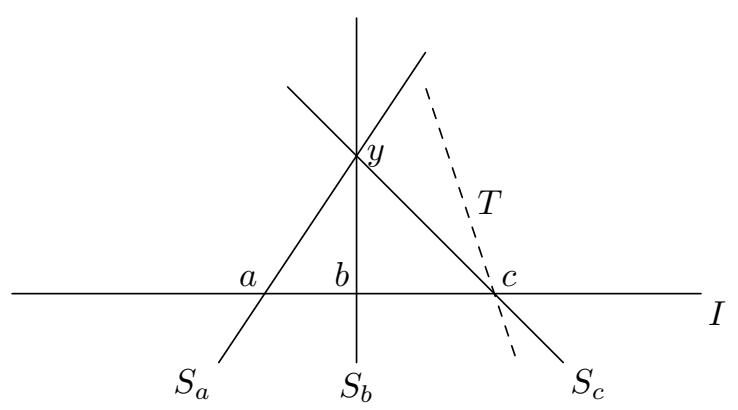

Figure 3: Sub-subcase 2.2.2.

\subsubsection{Every two segments intersect.}

See Figure 3. Let $p$ be a line containing at least two segments. Considering $p$ as a horizontal line, let $L$ be the segment whose right endpoint is the leftmost point among all right endpoints of the segments lying on $p$. Similarly, let $R$ be the segment whose left endpoint is the rightmost point among all left endpoints of the segments lying on $p$. Then $I=L \cap R$ is a non-empty interval (which may be degenerate), which is contained in all the segments lying on $p$. Let oblique segment be a segment not lying on $p$. According to the assumption of this sub-subcase, every oblique segment crosses $p$ somewhere in the interval $I$. Let $X$ be the set of all intersections of some oblique segment and $I$. If $X$ is empty, then an arbitrary point from $I$ pierces $\mathcal{S}$. If $|X|=1$ or $|X|=2$, then every segment contains at least one point from $X$. We are left with the case $|X| \geq 3$. Let $a, b, c$ be three different points from $X$ and let $S_{a}, S_{b}, S_{c}$ be some oblique segments such that $a \in S_{a}, b \in S_{b}, c \in S_{c}$. Among the segments $L, S_{a}, S_{b}, S_{c}$, the triple $S_{a}, S_{b}, S_{c}$ is the only one which can have a non-empty intersection. So there exists a point $y$ not lying on $p$ such that $\{y\}=S_{a} \cap S_{b} \cap S_{c}$. If every oblique segment contains $y$, we can choose $y$ and an arbitrary point from $I$ as the required two piercing points. In the other case there exists an oblique segment $T$ not containing $y$. The segment $T$ contains at most one point from $\{a, b, c\}$, so we can assume that $a, b \notin T$. But then, among the segments $L, S_{a}, S_{b}, T$ no three have a point in common, which is a contradiction with $(4,3)$-property.

\section{Discs}

Let $\mathcal{D}$ be a set of all closed unit discs in $\mathbb{R}^{2}$.

Theorem 5. $M_{\mathcal{D}}=3$.

Proof. First we establish the upper bound. 


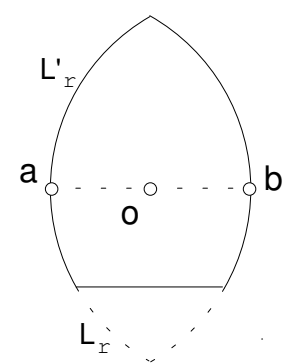

Figure 4: Case 1, regions $L_{r}$ and $L_{r}^{\prime}$.

Let $\mathcal{S}$ be a finite subcollection of $\mathcal{D}$ satisfying $(4,3)$-property. We will show that $P(\mathcal{S}) \leq 3$.

Let $C$ be the set of all centers of discs in $\mathcal{S}$. According to Lemma 3 we can use the following dual form of $(4,3)$-property: among every four points in $C$ some three can be covered by a closed unit disc. To prove that $P(\mathcal{S}) \leq 3$, it suffices to show that $C$ can be covered by three unit discs.

Let $r$ be the largest distance between two points of $C$. We will distinguish three cases:

1. $r \leq 2$ (every two discs from $\mathcal{S}$ have a non-empty intersection).

Let $a, b \in C$ be the points whose distance is equal to $r$. Then all points from $C$ lie in the lens-like region $L_{r}$, which is the intersection of two closed discs with the radius $r$ centered at the points $a, b$; see Figure 4 . Let $x$ be the line determined by points $a, b$. Consider $x$ as an $x$-axis of a Cartesian coordinate system with the origin $o$ at the center of the segment $a b$, such that $b$ has a positive $x$-coordinate. Let $\left(x_{1}, y_{1}\right) \in C$ be a point with the largest $y$-coordinate, and $\left(x_{2}, y_{2}\right) \in C$ a point with the least $y$-coordinate. Then $y_{1} \geq 0, y_{2} \leq 0$ and $y_{1}-y_{2} \leq r$. Without loss of generality, suppose that $\left|y_{1}\right| \geq\left|y_{2}\right|$. Then $y_{2} \geq-\frac{r}{2}$, so all the points from $C$ lie in the set $L_{r}^{\prime}=\left\{(x, y) \in L_{r} ; y \geq-\frac{r}{2}\right\}$. It now suffices to prove that $L_{r}^{\prime}$ can be covered by three closed unit discs. To finish the proof, we refer to case $3(2<r \leq \sqrt{8})$, where a set larger then $L_{r}^{\prime}$ is covered by three unit discs.

2. $r>\sqrt{8}$.

Let $A, B \in \mathcal{S}$ be the discs with the centers $a, b$ such that $|a-b|=r$. The $\operatorname{discs} A$ and $B$ are disjoint, so, according to Lemma 2 and by the symmetry, we can assume that all the discs from $\mathcal{S} \backslash\{A\}$ intersect $B$. By $(4,3)$-property and Helly's Theorem, all the discs from $\mathcal{S}$ disjoint with $A$ have a common point $b_{0} \in B$ (if there are just two such discs, use Lemma 2). Equivalently, all the points from $C$ whose distance from $a$ is larger than 2 can be covered by one closed unit disc centered at $b_{0}$. It remains to cover the set $C^{\prime}=\{s \in C ;|s-a| \leq 2\}$ by two closed unit discs. Except of $a$, every other point $s \in C^{\prime}$ satisfies $|s-b| \leq 2$, so it lies in the region $L$, which is the intersection of two closed discs of radius 2 centered at points $a, b$; see Figure 5 . Let $C^{\prime \prime}=C^{\prime} \backslash\{a\}$. If $\left|C^{\prime \prime}\right| \leq 1$, then $C^{\prime}$ contains at most two points and can be covered 


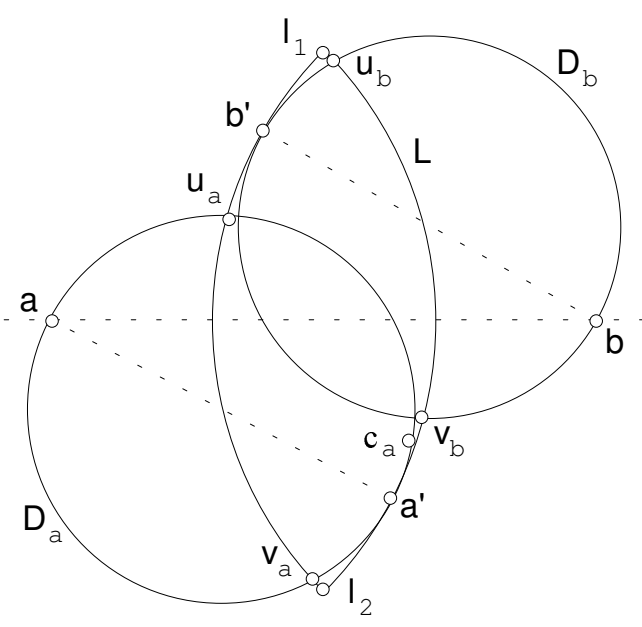

Figure 5: Case 2, discs $D_{a}$ and $D_{b}$ cover all the points from $C^{\prime}$.

by at most two closed unit discs. For the rest of this case suppose that $\left|C^{\prime \prime}\right| \geq 2$. By $(4,3)$-property, for every two points $c_{1}, c_{2} \in C^{\prime \prime}$ there exists either a closed unit disc containing points $a, c_{1}, c_{2}$ or a closed unit disc containing points $b, c_{1}, c_{2}$. Moreover, we can suppose that this disc has the point $a$ (or $b$ ) on its boundary:

Since $r>\sqrt{8}$, for every point $c \in L$ the sizes of the angles $b a c$ and $a b c$ are less than $\frac{\pi}{4}$. Thus for every two points $c_{1}, c_{2} \in L$ the angles $c_{1} a c_{2}$ and $c_{1} b c_{2}$ are acute. The rest follows from the following fact.

Lemma 6. Let $D$ be the smallest disc containing a triangle xyz. If the angle xyz is acute, then $y$ lies on the boundary of $D$.

Proof. Clearly, if $T=x y z$ is an obtuse or a right triangle with the longest side $x y$, then $D$ has the segment $x y$ as its diameter. If $T$ is acute, then $D$ is the disc whose boundary is the circumcircle of $T$.

Consider the same coordinate system as in case 1 and denote by $l_{1}, l_{2}$ the points of $L$ with the largest and the least $y$-coordinate. We define two discs, $D_{a}$ and $D_{b}$, which cover all the points from $C^{\prime}$. The discs $D_{a}$ and $D_{b}$ will be determined by their diameters $a a^{\prime}$ and $b b^{\prime}$.

Let $D$ be a closed unit disc with diameter $a a_{1}$, such that $D$ has a non-empty intersection with $L$. Then $a_{1}$ lies on the semicircle $S_{a}=\left\{z=\left(z_{x}, z_{y}\right) \in \mathbb{R}^{2} ;|z-a|=\right.$ $\left.2, z_{x}>-\frac{r}{2}\right\}$. If $a_{1} \in L \backslash\left\{l_{1}, l_{2}\right\}$, then the set $L \backslash D$ consists of two connected regions: the upper region $\bar{D}$ and the lower region $\underline{D}$.

In the other case $L \backslash D$ consists of a single connected region: either $\bar{D}$, if $a_{1}$ has a negative $y$-coordinate, or $\underline{D}$ otherwise. We define an upper boundary of $D$ as an arc, which is a common boundary of $D$ and $\bar{D}$ (in case that $a_{1}=l_{1}$ we define the upper boundary as $\left\{l_{1}\right\}$ ). Similarly we define a lower boundary of $D$. For the points of the 
semicircle $S_{a}$ we consider a "vertical" linear ordering: if $u=\left(u_{x}, u_{y}\right), v=\left(v_{x}, v_{y}\right) \in$ $S_{a}$ and $u_{y}<v_{y}$, we write $u \prec v$ and say that $u$ is lower than $v$, or equivalently, that $v$ is higher than $u$. We make similar definitions also for the discs having point $b$ on the boundary.

Suppose that no closed unit disc with $a$ or $b$ on its boundary covers all the points from $C^{\prime \prime}$. Consider the set $M_{a}$ of all closed unit discs $D$ with diameter $a a_{1}$ such that $a_{1}$ lies on the semicircle $S_{a}$ and the boundary of $D$ contains at least one point from $C^{\prime \prime}$. Symmetrically we define the set $M_{b}$. Let

$$
\left.M=\left\{\left(D_{a}^{\prime}, D_{b}^{\prime}\right) \in M_{a} \times M_{b} ; \overline{D_{a}^{\prime}} \cap\left(C^{\prime \prime} \backslash D_{b}^{\prime}\right)=\emptyset \wedge\left(C^{\prime \prime} \backslash D_{a}^{\prime}\right) \cap \underline{D_{b}^{\prime}}=\emptyset\right\}\right\} .
$$

Clearly, $M$ is a non-empty finite set. We choose the pair $\left(D_{a}, D_{b}\right)$ as a maximal element of $M$ according to the following partial order:

For the discs $D_{a}^{1}, D_{b}^{1}, D_{a}^{2}, D_{b}^{2}$ with the diameters $a a_{1}, a a_{2}, b b_{1}, b b_{2}$, we put $\left(D_{a}^{1}, D_{b}^{1}\right) \ll$ $\left(D_{a}^{2}, D_{b}^{2}\right)$ if and only if

$$
\left(a_{1} \succeq a_{2} \wedge b_{1} \prec b_{2}\right) \vee\left(a_{1} \succ a_{2} \wedge b_{1} \preceq b_{2}\right) .
$$

The discs $D_{a}$ and $D_{b}$ cover $C^{\prime \prime}$ if and only if $\left(\overline{D_{a}} \cup \underline{D_{a}}\right) \cap\left(\overline{D_{b}} \cup \underline{D_{b}}\right) \cap C^{\prime \prime}=\emptyset$. By the definition of $M$, it suffices to prove that $\underline{D_{a}} \cap \overline{D_{b}} \cap C^{\prime \prime}=\emptyset$.

From the maximality of $\left(D_{a}, D_{b}\right)$ it follows that there exists a point $c_{a} \in C^{\prime \prime} \backslash D_{b}$ on the upper boundary of $D_{a}$ and a point $c_{b} \in C^{\prime \prime} \backslash D_{a}$ on the lower boundary of $D_{b}$. It cannot happen both $c_{a} \in \overline{D_{b}}$ and $c_{b} \in \underline{D_{a}}$, since in this case there exists no closed unit disc with $a$ or $b$ on its boundary covering both points $c_{a}$ and $c_{b}$. Thus, without loss of generality, we can suppose that $c_{a} \in \underline{D_{b}}$.

For contradiction, suppose that $\underline{D_{a}}$ and $\overline{D_{b}}$ have a non-empty intersection. Let $u_{a}$ and $v_{a}$ be the intersections of the boundary of $D_{a}$ with the semicircle $S_{b}$, such that $u_{a}$ is higher than $v_{a}$. Similarly we define $u_{b}$ and $v_{b}$ as the intersections of the boundary of $D_{b}$ with the semicircle $S_{a}$, so that $u_{b}$ is higher than $v_{b}$. The region $D_{a}$ is bounded by three $\operatorname{arcs} v_{a} a^{\prime}, a^{\prime} l_{2}, l_{2} v_{a}$, and $\overline{D_{b}}$ is bounded by the arcs $u_{b} b^{\prime}, b^{\prime} l_{1}$, $l_{1} u_{b}$. If $b^{\prime}$ were higher than $v_{a}$ and $u_{b}$ higher than $a^{\prime}$, the regions $\underline{D_{a}}$ and $\overline{D_{b}}$ would be disjoint (they would be separated by the line $v_{a} a^{\prime}$, for example). Since there is a point $c_{a} \in \underline{D_{b}}$ on the upper boundary of $D_{a}$, either $b^{\prime}$ is higher than $u_{a}$ or $v_{b}$ is

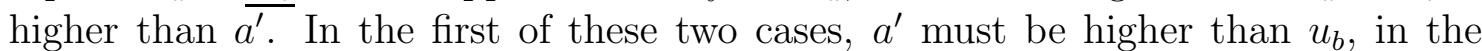
second case $b^{\prime}$ must be lower than $v_{a}$. Since these cases are symmetric, it suffices to consider only the first case.

We observe that $u_{a}$ has larger $y$-coordinate than $a^{\prime}$ : consider a horizontal line $x_{a^{\prime}}$ going through the point $a^{\prime}$. It crosses the boundary of $D_{a}$ at the point $t_{a}$ with the $x$-coordinate equal to $-\frac{r}{2}$ (the angle $a^{\prime} t_{a} a$ is right). It implies that $t_{a}$ lies outside the region $L$ and that the line $x_{a^{\prime}}$ crosses $S_{b}$ (the left part of the boundary of $L$ ) at a point which lies inside $D_{a}$, thus lower than $u_{a}$. Similarly we get that $u_{b}$ has larger $y$-coordinate than $b^{\prime}$, which contradicts the assumptions that $b^{\prime}$ is higher than $u_{a}$ and $a^{\prime}$ higher than $u_{b}$. 


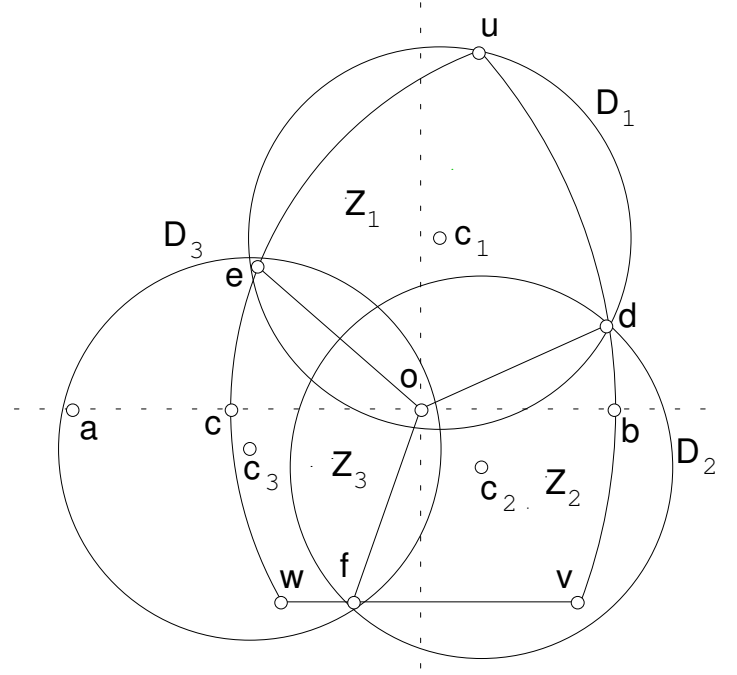

Figure 6: Case 3 , discs $D_{1}, D_{2}, D_{3}$ cover the region $L_{r}^{\prime \prime}$.

3. $2<r \leq \sqrt{8}$.

See Figure 6.

As in case 2 , let $A, B \in \mathcal{S}$ be the discs with the centers $a, b$ such that $|a-b|=r$ and all the discs from $\mathcal{S}$ except of $A$ intersect $B$. Let $C^{\prime}=C \backslash\{a\}$. All the points from $C^{\prime}$ lie in the area $L_{r}=\left\{z \in \mathbb{R}^{2} ;|z-a| \leq r,|z-b| \leq 2\right\}$. Hence it is sufficient to cover the set $L_{r} \cup S_{r}$, where $S_{r}$ is the segment $a b$, with three closed unit discs. Consider a Cartesian coordinate system such that $b=(1,0)$, and $a=(1-r, 0)$. By Lemma 2, every two discs from $\mathcal{S} \backslash\{A\}$ have a non-empty intersection, so every two points from $C^{\prime}$ have a vertical distance at most 2. Thus, without loss of generality, all the points from $C^{\prime}$ have $y$-coordinate at least -1 . So it suffices to cover the set $L_{r}^{\prime \prime}=L_{r}^{\prime} \cup S_{r}$, where $L_{r}^{\prime}=\left\{z=\left(x_{z}, y_{z}\right) ; z \in L_{r}, y_{z} \geq-1\right\}$. Without loss of generality, we can assume $r=\sqrt{8}$. It is easy to see that $L_{\sqrt{8}}^{\prime \prime}$ covers every set $L_{r}^{\prime}$ defined in case $1(r \leq 2)$.

We will divide $L_{r}^{\prime \prime}$ into three sets $Z_{1}, Z_{2}, Z_{3}$ and we cover each of them by one unit disc. Let $u=\left(\frac{2-\sqrt{2}}{2}, \frac{\sqrt{14}}{2}\right), v=(1-\sqrt{8}+\sqrt{7},-1), w=(1-\sqrt{3},-1)(u, v$ and $w$ are the boundary vertices of the region $\left.L_{r}^{\prime}\right), o=(0,0), c=(-1,0), d=(1-$ $\left.\sqrt{8}+\sqrt{8-0.43^{2}}, 0.43\right), e=\left(1-\sqrt{4-0.75^{2}}, 0.75\right)$, and $f=(-0.35,-1)$. The points $d, e, f$ lie on the boundary of $L_{r}^{\prime}$ and the segments od, oe, of divide $L_{r}^{\prime \prime}$ into three parts $Z_{1}=$ odue, $Z_{2}=o f v d$, and $Z_{3}=o e w f \cup a c$. For $i=1,2,3$, part $Z_{i}$ is covered by the closed unit disc $D_{i}$ centered at $c_{i}$, where $c_{1}=(0.1,0.9), c_{2}=(0.3,-0.3)$, and $c_{3}=(-0.9,-0.2)$ : it is straightforward to check that $o, d, u, e \in D_{1}, o, f, v, d \in D_{2}$ and $o, e, w, f, a, c \in D_{3}$, which implies $Z_{i} \subseteq D_{i}$ for $i=1,2,3$ (the boundary curves of the sets $Z_{i}$ consist of segments or arcs which have less curvature than the unit circle).

The lower bound is established by the example of Grünbaum [5], who constructed nine 


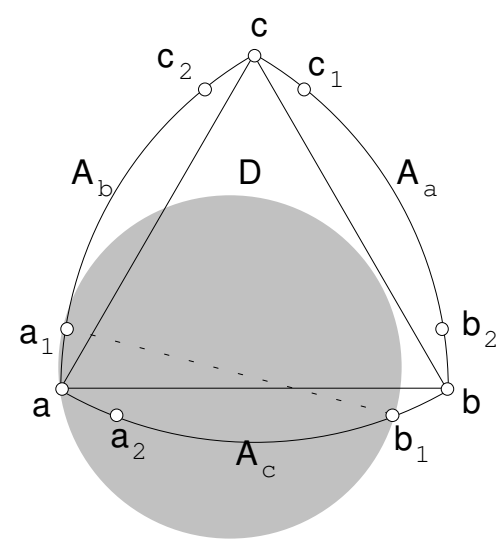

Figure 7: Nine points of $S$ and the disc $D$ covering points $b_{1}, a_{1}, a$.

pairwise intersecting unit discs that cannot be pierced by two points. For completeness, we describe the construction here (at least we need to show that the $(4,3)$-property is satisfied). We construct a nine-point set $S=\left\{a, b, c, a_{1}, a_{2}, b_{1}, b_{2}, c_{1}, c_{2}\right\}$ in the plane, which cannot be covered by two closed unit discs and satisfies the dual version of $(4,3)$ property. See Figure 7.

Let $a, b, c$ be the vertices of an equilateral triangle with side of length 2 . Let $A_{a}$ be the arc of the circle with center $a$ and radius 2, with endpoints $b, c$ and central angle $\frac{\pi}{3}$. Similarly arcs $A_{b}$ and $A_{c}$ are defined. In other words, the points $a, b, c$ and the arcs $A_{a}, A_{b}, A_{c}$ form the vertices and edges of a Reuleaux triangle of width 2 . Let $\varepsilon>0$ be sufficiently small. Let $a_{1}, c_{2} \in A_{b}, b_{1}, a_{2} \in A_{c}, c_{1}, b_{2} \in A_{a}$ be such points, that $\left|a_{1}-a\right|=\left|a_{2}-a\right|=\left|b_{1}-b\right|=\left|b_{2}-b\right|=\left|c_{1}-c\right|=\left|c_{2}-c\right|=\varepsilon$.

Now we verify that both required conditions are satisfied:

- Suppose that $S$ can be covered by two closed unit discs. Then one of them, $D_{1}$, must cover two points from the set $\{a, b, c\}$, say $a$ and $b$. The distance between $a$ and $b$ is equal to the length of the diameter of the unit disc. Hence, $D_{1}$ is centered at the center of the segment $a b$. Arcs $A_{a}$ and $A_{b}$ intersect $D_{1}$ only at points $a$ and $b$, so $D_{1}$ covers only points $a, b, a_{2}$ and $b_{1}$. But points $c, a_{1}$ and $b_{2}$ obviously cannot be covered by a closed unit disc (the circle circumscribed to the acute triangle $c a_{1} b_{2}$ has radius almost $\frac{2 \sqrt{3}}{3}$ ), which is a contradiction.

- Let $Q \subset S$ be a four-point set. Without loss of generality, suppose that $Q$ contains at least two points from the set $\left\{a, a_{1}, a_{2}\right\}$. If $Q$ contains all these three points, then $\left\{a, a_{1}, a_{2}\right\} \subset Q$ is a triple which is covered by a closed unit disc centered at $a$. Otherwise, $Q$ contains exactly two of the points $a, a_{1}, a_{2}$. By symmetry, we can suppose that $a_{1} \in Q$.

Suppose that $a \in Q$. The quadruple $Q$ contains at least one point from the set $R=\left\{c_{2}, c, c_{1}, b_{2}, b_{1}\right\}$. Hence it suffices to prove that every triple $\left\{a_{1}, a, x\right\}$, where $x \in R$, can be covered with a closed unit disc. If $x \in\left\{c_{2}, c, c_{1}, b_{2}\right\}$, then $\left\{a_{1}, a, x\right\}$ is covered by the disc with the diameter $a x$, since $|a-x| \leq 2$ and the angle 
$a a_{1} x$ is obtuse. The remaining triple $\left\{a_{1}, a, b_{1}\right\}$ is covered by the disc $D$ with the diameter $a_{1} b_{1}$ : let $o$ be the circumcenter of the triangle $a b c$. Then triangles $o a_{1} b_{1}$ and $o a b$ are similar and $\left|o-b_{1}\right|<|o-b|$, which implies $\left|a_{1}-b_{1}\right|<2$, so $D$ has a radius less than 1 . The disc $D$ covers also the point $a$, since $a_{1} a b_{1}$ is a right triangle: $\left|\varangle b_{1} a a_{1}\right|=\left|\varangle b a a_{1}\right|+\left|\varangle b_{1} a b\right|=\left|\varangle c b b_{1}\right|+\left|\varangle b_{1} a b\right|=\frac{\pi}{3}+\left|\varangle a b b_{1}\right|+\left|\varangle b_{1} a b\right|=\frac{\pi}{3}+\frac{\pi}{6}=\frac{\pi}{2}$.

Suppose that $a_{2} \in Q$. Then all the triples $\left\{a_{1}, a_{2}, x\right\}$, where $x \in\left\{c_{2}, c, c_{1}, b_{2}, b, b_{1}\right\}$, can be covered by a closed unit disc: by symmetry, it suffices to prove that only for $x \in\left\{c_{2}, c, c_{1}\right\}$. In that case, $\left\{a_{1}, a_{2}, x\right\}$ is covered by the disc with the diameter $a_{2} x$, since $\left|a_{2}-x\right| \leq 1$ and the angle $a_{2} a_{1} x$ is obtuse.

\section{$5 \quad$ Triangles}

In this section we analyze the collections of positively homothetic triangles.

By applying an appropriate affine transformation, we can assume without loss of generality that all the considered triangles are equilateral. Let $T_{\lambda} \subset \mathbb{R}^{2}$ be an equilateral triangle defined as a convex hull of points $(0,0),(\lambda, 0)$ and $\left(\frac{\lambda}{2}, \frac{\sqrt{3} \lambda}{2}\right)$. Let $\mathcal{T}$ be a collection of all triangles $T_{\lambda}+v$, where $\lambda>0$ and $v \in \mathbb{R}^{2}$ is an arbitrary vector. Let $\mathcal{T}_{1} \subset \mathcal{T}$ be a collection of all unit triangles from $\mathcal{T}$, i. e., the triangles with $\lambda=1$. Our aim is to show that $M_{\mathcal{T}}=M_{\mathcal{T}_{1}}=3$.

For $T=T_{\lambda}+v \in \mathcal{T}$, the line determined by points $(0,0)+v$ and $(\lambda, 0)+v$ is called the bottom line of $T$. Similarly, the right line is determined by points $(\lambda, 0)+v$ and $\left(\frac{\lambda}{2}, \frac{\sqrt{3} \lambda}{2}\right)+v$ and $(0,0)+v$, and the left line is determined by points $(\lambda, 0)+v,\left(\frac{\lambda}{2}, \frac{\sqrt{3} \lambda}{2}\right)+v$ and $(0,0)+v,\left(\frac{\lambda}{2}, \frac{\sqrt{3} \lambda}{2}\right)+v$. The point $\left(\frac{\lambda}{2}, \frac{\sqrt{3} \lambda}{2}\right)+v$ is called the top of the triangle $T_{\lambda}+v$.

Now we present the upper and the lower bound for the maximum piercing number of positively homothetic triangles.

Theorem 7. $M_{\mathcal{T}} \leq 3$.

Proof. As usual, let $\mathcal{S}$ be a finite subcollection of $\mathcal{T}$ satisfying $(4,3)$-property. We will show that $P(\mathcal{S}) \leq 3$.

Let $T \in \mathcal{S}$ be the triangle which has the largest $y$-coordinate of its bottom line. If $T$ is not unique, pick one such triangle arbitrarily. Denote by $a, b, c$ the vertices of $T$ so that $a b$ is the bottom line of $T$. Without loss of generality, we may assume that $T=T_{1}$, i. e., $a=(0,0)$ and $b=(1,0)$. If $S \in \mathcal{S}$ and $S \cap T$ is non-empty, then the intersection of the segment $a b$ and $T$ is a non-empty segment (which may be degenerate). Let $l_{S}$ and $r_{S}$ be the $x$-coordinates of the left and the right endpoint of this segment.

Consider several cases:

1. The triangle $T$ does not intersect any other triangle.

Let $U, V, W$ be different elements of $\mathcal{S} \backslash\{T\}$. Then, according to the (4,3)-property for the quadruple $T, U, V, W$, the triple $U, V, W$ has a non-empty intersection. 

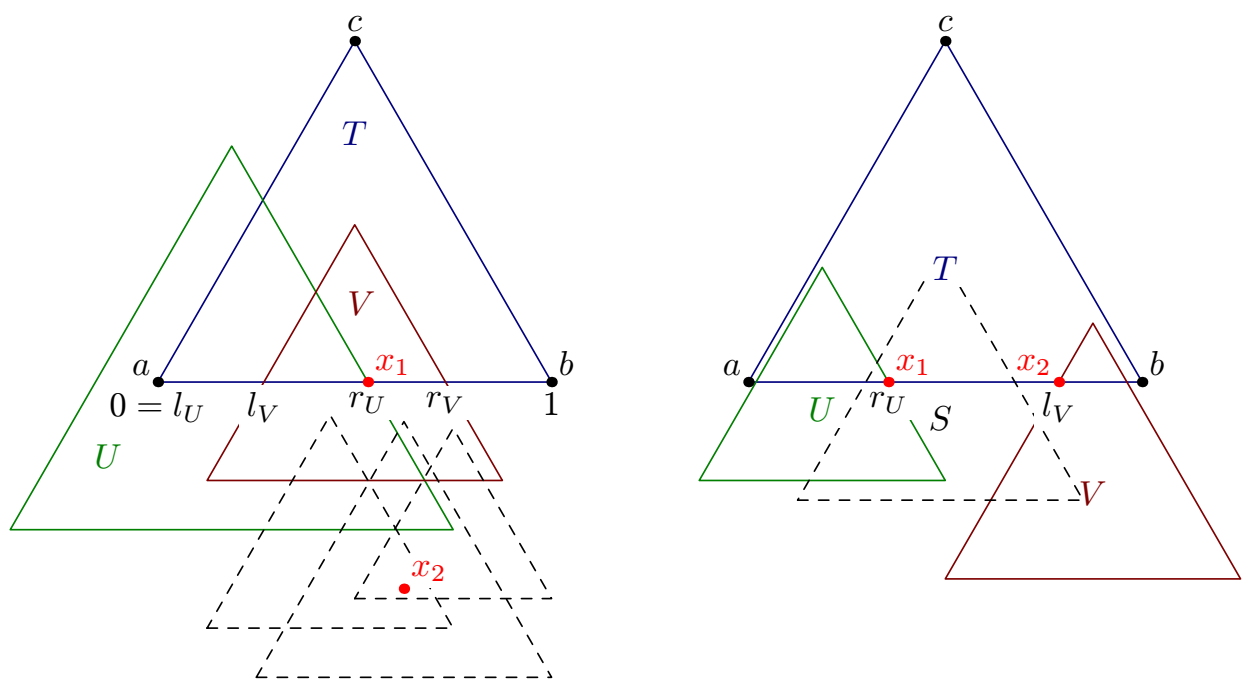

Figure 8: Subcases 2.1 and 2.2.

The assumption $|\mathcal{T}| \geq 4$ implies that $|\mathcal{S} \backslash\{T\}| \geq 3$. Hence, by Helly's Theorem, the intersection of $\mathcal{S} \backslash\{T\}$ is non-empty. Then arbitrary two points $x_{1} \in \bigcap(\mathcal{S} \backslash\{T\})$ and $x_{2} \in T$ pierce $\mathcal{S}$.

2. The triangle $T$ intersects some other triangle.

Let $U \in \mathcal{S}$ be the triangle such that $r_{U}$ is the smallest possible. Similarly, let $V \in \mathcal{S}$ be the triangle such that $l_{V}$ is the largest possible. We distinguish three subcases. See Figure 8 for the first two of these subcases.

2.1. $r_{U} \geq l_{V}$.

Let $x_{1}=\left(r_{U}, 0\right)$. If $S \in \mathcal{S}$ is a triangle intersecting $T$, then $r_{S} \geq r_{U}$ and $l_{S} \leq l_{V} \leq r_{U}$, hence $x_{1} \in S$. As in case 1 , there exists a point $x_{2}$ which is in the intersection of triangles not intersecting $T$ (if there are at most two such triangles, they can be pierced by two points $x_{2}$ and $x_{3}$ ).

2.2. $r_{U}<l_{V}$ and $U \cap V$ is empty.

Let $x_{1}=\left(r_{U}, 0\right)$ and $x_{2}=\left(l_{V}, 0\right)$. We will show that $\left\{x_{1}, x_{2}\right\}$ pierces $\mathcal{S}$. Clearly, $T$ and $U$ are pierced by $x_{1}$ and $V$ is pierced by $x_{2}$. Let $S \in \mathcal{S} \backslash\{T, U, V\}$. We will use (4,3)-property for the triangles $S, T, U, V$. Since $U \cap V=\emptyset$, we have $S \cap T \cap U \neq \emptyset$ or $S \cap T \cap V \neq \emptyset$. It means that $S \cap T \neq \emptyset$ and $l_{S} \leq r_{U} \leq r_{S}$ or $r_{S} \geq l_{V} \geq l_{S}$, which implies that $x_{1} \in S$ or $x_{2} \in S$.

2.3. $r_{U}<l_{V}$ and $U \cap V$ is non-empty.

Let $x_{1}=\left(r_{U}, 0\right)$ and $x_{2}=\left(l_{V}, 0\right)$. We will find a point $x_{3} \in U \cap V$ such that $\left\{x_{1}, x_{2}, x_{3}\right\}$ pierces $\mathcal{S}$. Let $\mathcal{M}=\{S \in \mathcal{S} \mid S \cap T=\emptyset\}$ and $\mathcal{Z}=\mathcal{M} \cup\{U, V\}$. First, we establish that $\bigcap \mathcal{Z} \neq \emptyset$. If $\mathcal{Z}=\{U, V\}$, then $\bigcap \mathcal{Z} \neq \emptyset$ from the assumption of this subcase. If $|\mathcal{Z}| \geq 3$, then it is sufficient to show that Helly's property from Theorem 1 is satisfied. Let $X, Y, Z \in \mathcal{Z}$. According to the 

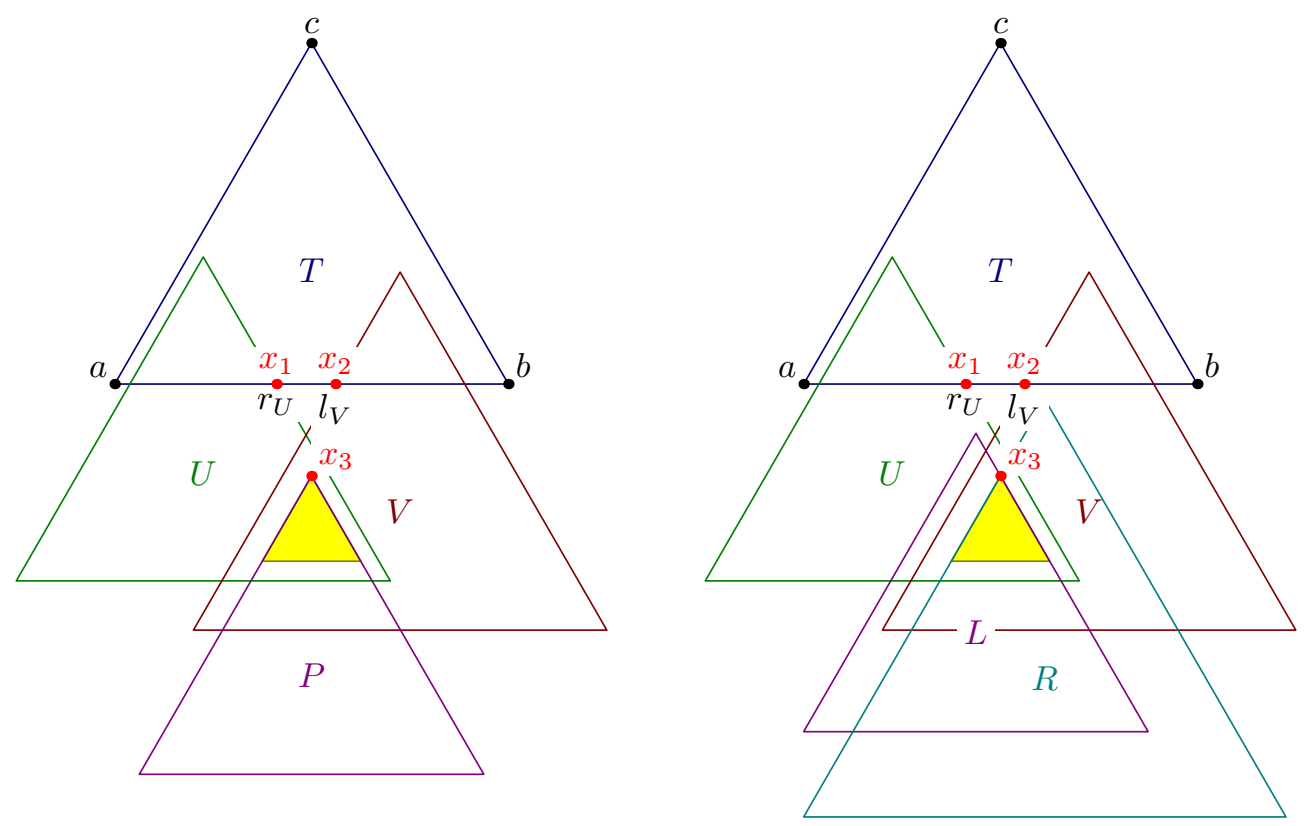

Figure 9: Sub-subcases 2.3.1 and 2.3.2.

(4,3)-property for the quadruple $X, Y, Z, T$ we obtain that $X \cap Y \cap Z$ is non-empty (by an easy discussion of three cases according to the cardinality of $\{X, Y, Z\} \cap\{U, V\})$. Hence Helly's property is satisfied. It is easy to see that $\bigcap \mathcal{Z}$ is a triangle or a point. In the first case pick $x_{3}$ as the top of that triangle, in the second case pick $x_{3}$ such that $\left\{x_{3}\right\}=\bigcap \mathcal{Z}$. It remains to show that every triangle $S \in \mathcal{S}$ is pierced by some of the points $x_{1}, x_{2}, x_{3}$. We know this for $S \in\{T, U, V\}$ and for every triangle $S \in \mathcal{S}$ disjoint with $T$. Now, let $S \neq T, U, V$ be a triangle from $\mathcal{S}$ intersecting $T$. If $l_{S} \leq r_{U}$, then $x_{1} \in S$. If $r_{S} \geq l_{V}$, then $x_{2} \in S$.

Suppose that $r_{U}<l_{S} \leq r_{S}<l_{V}$. We will show that $x_{3} \in S$. We have $T \cap U \cap S=\emptyset$ and $T \cap V \cap S=\emptyset$. Now, distinguish two sub-subcases.

2.3.1. There exists $P \in \mathcal{Z}$ such that $x_{3}$ is a top of $P$.

Then $P \neq U, V$, since the tops of $U$ and $V$ are above the bottom line of $T$. Use the (4,3)-property for the quadruple $S, T, U, P$. We know that $T \cap P=\emptyset$ and $S \cap T \cap U=\emptyset$, hence $S \cap U \cap P \neq \emptyset$. In particular, $S \cap P \neq \emptyset$. On the other hand, $x_{3}$ is below the left line of $V$, which is below the left line of $S\left(l_{S}<l_{V}\right)$. Similarly, $x_{3}$ is below the right line of $S$. Thus $S \cap P \neq \emptyset$ implies that $x_{3} \in S$.

2.3.2. There are two distinct triangles $L, R \in \mathcal{Z}$ such that $x_{3}$ is on the left line of $R$ and on the right line of $L$.

Then the statement easily follows from the $(4,3)$-property for the triangles $S, T, L$ and $R$.

We have discussed all the cases so the proof is finished. 


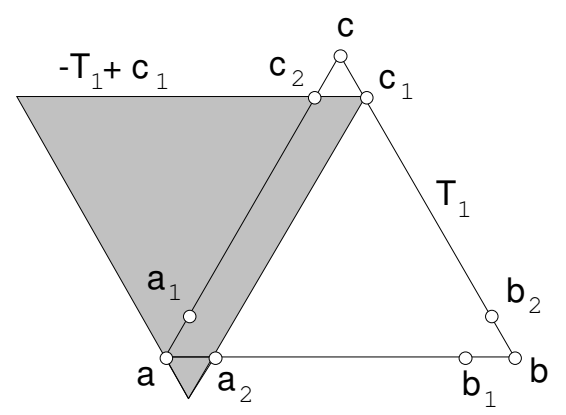

Figure 10: Nine points of $S$ and the triangle $-T_{1}+c_{1}$.

Theorem 8. $M_{\mathcal{T}_{1}} \geq 3$.

Proof. Similarly as in the case of unit discs, we construct a nine-point set $S=\{a, b, c$, $\left.a_{1}, a_{2}, b_{1}, b_{2}, c_{1}, c_{2}\right\}$ which cannot be covered by two translated copies of $-T_{1}$ and satisfies a dual version of the (4,3)-property: among every four points from $S$ some three can be covered by a translated copy of $-T_{1}$. See Figure 10 .

Let $a=(0,0), b=(1,0), c=\left(\frac{1}{2}, \frac{\sqrt{3}}{2}\right)$ be the vertices of the equilateral triangle $T_{1}$. We put the remaining six points on the sides $a b, b c, c a$ of this triangle: let $a_{1}, c_{2} \in c a$, $b_{1}, a_{2} \in a b, c_{1}, b_{2} \in b c$ be such points, that $\left|a_{1}-a\right|=\left|a_{2}-a\right|=\left|b_{1}-b\right|=\left|b_{2}-b\right|=$ $\left|c_{1}-c\right|=\left|c_{2}-c\right|=\varepsilon$, where $\varepsilon>0$ is sufficiently small.

Suppose that $S$ can be covered by two translated copies of $-T_{1}$. Then one of these two copies, $T^{\prime}$, covers two of the points $a, b, c$. By symmetry, we can assume that $a, b \in T^{\prime}$. Then $a$ and $b$ are two vertices of $T^{\prime}$ and $T^{\prime} \cap T=a b$, so the triangle $T^{\prime}$ covers only those four points from $S$ lying on $a b$. The remaining points have to be covered by the second translated copy of $-T_{1}$, but it is obviously impossible even for the three points $c, a_{1}, b_{2}$.

It remains to verify that $S$ satisfies the dual $(4,3)$-property. Let $Q$ be a four-point subset of $S$. Without loss of generality, we can assume that $Q$ contains exactly two points from the set $\left\{c, c_{1}, c_{2}\right\}$ and that $c_{2} \in Q$. If $a_{1} \in Q$ or $a_{2} \in Q$, then the triangle $-T_{1}+c+a_{2}$ (a translated copy of $-T_{1}$ with the bottom vertex at $a_{2}$ ) contains at least three points from $Q$, since it covers points $c, c_{1}, c_{2}, a_{1}$ and $a_{2}$. The case when $b_{1} \in Q$ or $b_{2} \in Q$ is symmetric. We are left with the case when $Q$ contains points $a, b, c_{2}$ and one of the points $c, c_{1}$. If $Q=\left\{a, b, c_{2}, c\right\}$, then points $a, c_{2}, c$ are covered by the triangle $-T_{1}+c$ with the bottom vertex at $a$. If $Q=\left\{a, b, c_{2}, c_{1}\right\}$, then the points $a, c_{2}, c_{1}$ are covered by the triangle $-T_{1}+c_{1}$ with the upper right vertex at $c_{1}$.

According to the inequality $M_{\mathcal{T}} \geq M_{\mathcal{T}_{1}}$, the previous two results imply the following corollary.

Theorem 9. $M_{\mathcal{T}}=M_{\mathcal{T}_{1}}=3$. 


\section{Acknowledgements}

We would like to thank to Pavel Valtr and Jan Kratochvíl who led the seminar under which this article has originated. We would also like to thank to Peter Bella, Josef Cibulka and Marek Tesař, who were participants of the seminar, for their notable comments.

\section{References}

[1] N. Alon and D. J. Kleitman, Piercing convex sets and the Hadwiger Debrunner (p,q)-problem, Advances in Mathematics 96 (1992), 103-112.

[2] N. Alon and D. J. Kleitman, A purely combinatorial proof of the Hadwiger Debrunner $(p, q)$-conjecture, Electr. J. Comb. 4(2) (1997), R1, 8pp.

[3] L. Danzer, Zur Lösung des Gallaischen Problems über Kreisscheiben in der Euklidischen Ebene (German), Studia Sci. Math. Hungar. 21 (1986), No. 1-2, 111-134.

[4] J. Eckhoff, A survey of the Hadwiger-Debrunner $(p, q)$-problem, Discrete and computational geometry, Algorithms Combin. 25, Springer, Berlin (2003), 347-377.

[5] B. Grünbaum, On intersections of similar sets, Portugal. Math. 18 (1959), 155-164.

[6] H. Hadwiger and H. Debrunner, Über eine variante zum Hellyschen satz (German), Arch. Math. 8 (1957), 309-313.

[7] H. Hadwiger and H. Debrunner, Kombinatorische Geometrie in der Ebene (German), Monographies de "L'Enseignement Mathématique", No. 2, Institut de Mathématiques, Université, Genève (1960).

[8] H. Hadwiger, H. Debrunner add V. Klee, Combinatorial geometry in the plane, Holt, Rinehart and Winston, New York (1964).

[9] E. Helly, Über Mengen konvexer Körper mit gemeinschaftlichen Punkten (German), Jahresber. Deutsch. Math. Verein. 32 (1923), 175-176.

[10] R. N. Karasev, Transversals for families of translates of a two-dimensional convex compact set, Discrete Comput. Geom. 24 (2000), No. 2-3, 345-353.

[11] D. J. Kleitman, A. Gyárfás and G. Tóth, Convex sets in the plane with three of every four meeting, Combinatorica 21(2) (2001), 221-232.

[12] L. Stachó, A solution of Gallai's problem on pinning down circles (Hungarian), Mat. Lapok 32 (1981/84), No. 1-3, 19-47.

[13] G. Wegner, Über Helly-Gallaische Stichzahlprobleme (German), 3. Kolloqium Über Diskrete Geometrie, Salzburg (1985), 277-282. 